\title{
Accessing the feasibility of monitoring small time-shifts due to geomechanical effects in a stiff reservoir
}

Vitor Leal de Mello', Diogo Folador Rossi' ${ }^{1}$, Ricardo Fernandes do Amaral ${ }^{1}$, André Bulcão', Guilherme Vasquez ${ }^{1}$, Yves-MarieLeroy $^{2}$, Vinicius Lemos de Almeida ${ }^{1}$, Jorg Herwanger ${ }^{3}$

${ }^{1}$ Petrobras, ${ }^{2}$ Total, ${ }^{3}$ MP-Geomechanics

Copyright 2021, SBGf - Sociedade Brasileira de Geofísica

This paper was prepared for presentation during the $17^{\text {th }}$ International Congress of the Brazilian Geophysical Society held in Rio de Janeiro, Brazil, 16-19 August 2021.

Contents of this paper were reviewed by the Technical Committee of the $17^{\text {th }}$ International Congress of the Brazilian Geophysical Society and do not necessarily represent any position of the SBGf, its officers or members. Electronic reproduction or storage of any part of this paper for commercial purposes without the written consent of the Brazilian Geophysical Society is prohibited.

\section{Abstract}

This work presents the first results of recent efforts in modeling 4D seismic signals related to geomechanical effects in a pre-salt carbonate field, caused by reservoir production-induced rock properties changes in the reservoir and the overburden. The focus is to investigate the potential detectability of expected minute time-shifts in a stiff carbonate environment. Emphasis is placed on understanding the effect of the behavior of the salt-layer directly above the reservoir on the expected timeshifts. We examine the mechanical response of salt to production of the underlying reservoir, and how this mechanical response may affect observable time-lapse timeshifts.
\end{abstract}

We ask and answer two distinct questions from 3D geomechanical simulations during different stages of reservoir production into and calculation of $4 \mathrm{D}$ seismic travel-time differences. The first question assesses the robustness of 1D cross-correlation to measure sub-sample time-shifts. We first create a monitor survey by warping 3D seismic field data using traveltime differences derived from 3D geomechanical modelling, followed by application of a strain-sensitive rock-physics model. The maximum simulated timeshifts are approximately 0.2 to $0.3 \mathrm{~ms}$ at the top of the reservoir under the assumption of vertical wave propagation. We use the warped dataset and the original field dataset to measure the (known) timeshifts. We show that timeshifts of this magnitude can be measured in the presence of realistic amounts of coherent and random noise. The second question concerns the ability to measure time-lapse timeshifts after shot-receiver records went through a seismic processing sequence. Base- and monitor seismic data are generated from seismic velocity models using an ocean-bottom node (OBN) survey geometry. The velocity model for the monitor survey is again perturbed by the strain-induced velocity changes and both data are migrated using a least-squares reverse time migration (LSRTM). Time-lapse timeshifts are then analyzed from the final migrated images of the base- and monitor surveys.

The geomechanical response of the salt layer to production from pre-salt reservoirs is still poorly understood. We therefore geomechanically simulate two end-members, whereby (i) salt behaves elastically and can maintain differential stress over production time-scales and (ii) differential stress in salt is small, resulting in a near isotropic stress state inside salt. The two different scenarios of salt behavior cause a marked difference in the post-production stress fields and a distinct response in terms of magnitude and shape of 4D time-shift anomalies. This means that the ability of monitoring seismic velocity changes in the overburden may result in an indication of how salt layers behave over production time, and map regions where stresses and strains create a risk to wellbore integrity and to fault reactivation.

\section{Introduction and Contextualization}

The issue of subsidence induced by oil and gas production affecting surface infrastructure is known by the industry at least since the beginning of the last century. Documented examples include the Goose Creek and the Wilmington Fields, both in U.S., (e.g., Nagel, 2001 and Mayuga, 1970), several oil fields bordering Lake Maracaibo in Venezuela (e.g., Geertsma, 1973), and the Ekofisk and Valhall giant chalk fields in the North Sea (e.g., Sulak, 1991). All these examples displayed several meters of vertical surface displacement and/or experienced differential horizontal displacements compromising the infrastructure of neighborhood areas or the production facilities. The costs to maintain and repair infrastructure in each case is in the 100 s of millions to low billions of USD.

Surface subsidence is arguably the most visible expression of geomechanical effects due to reservoir production. However, other geomechanical effects can also have detrimental consequences on the ability to operate fields in a safe and cost-efficient manner. At the giant Groningen gas field in the Netherlands the occurrence of microseismic events, which could be felt at the surface, has led to the decision to cease production before the reservoir is fully depleted. Stress changes can lead to re-activation of faults, slip along weak bedding planes, or large shear stress at interfaces of layers with large material property contrasts. All three effects have been associated with shear failure of existing well stock (Dusseault et al., 2001). Clearly, the consequences of such events go beyond the huge economic impact, but also affect relations with society and investors and cause environmental damages. Therefore, constant attention about the safety of operations through geomechanical studies has become an important part of field development planning. Establishing safe operations margins contributes to maximizing oil recovery at the same time as mitigating risks. Due to 
uncertainties inherent in all subsurface models, predictions of geomechanical effects are also uncertain. 4D seismic monitoring is an important tool to calibrate 4D geomechanical models, and monitor stress changes over time to identify initial stages of deformation in the reservoir and surrounding rocks.

According to Geertsma (1973), the combination of high rock compressibility, thick production intervals and large pressure depletion, all contribute to reservoir compaction and subsidence. In the Brazilian pre-salt carbonate reservoirs, the only criteria that does not satisfy the conditions for large amounts of compaction is low rock compressibility of the rock matrix. However, the presence of fractures and karsts might create softer reservoirs than the compressibility of the intact rock suggests. Moreover, during basin evolution halokinesis induced sets of faults, which occasionally reach the sea bottom, represent an additional risk of fault reactivation. The behavior of evaporites under stress along production time is unknown in the context of reservoir engineering, since pre-salt production has only just started, and simulations are not yet accurate enough to be predictive without calibration to field experience. Thus, this uncertainty must be considered when evaluating the potential effects of production on reservoir and surrounding rocks.

Time-lapse seismic applied to monitoring geomechanical changes was shown to be feasible using field case-studies from a range of geological settings by Hatchell and Bourne (2005). The integration and calibration of 4D geomechanical models with 4D seismic data is discussed in detail in Herwanger and Koutsabeloulis (2011). In a review paper, MacBeth et al., (2019) present an overview of reported time-shifts from worldwide 4D projects where observed time-shifts range from approximately 0.2 to 25 ms. Modelling of different geomechanical scenarios to study the effect on time-lapse seismic data was proposed in Herwanger and Horne (2009). Drawing on the work of these authors, we pose the following main questions guiding this work: what are the expected magnitudes of time-lapse timeshifts for the Brazilian pre-salt fields; can timeshifts of these magnitudes be measured; what is the effect of different scenarios of salt mechanical behavior on time-lapse seismic signals; and how may observations of such timeshifts be utilized to improve the quality of geomechanical models for field development?

The work presented in this paper serves three main purposes. Firstly, it describes the ongoing workflow developments at Petrobras and its partners to create a trained cadre of geoscientists and engineers able to apply and further develop techniques combing 4D seismic and 4D geomechanics for safe and cost-efficient field development. Secondly, it aims to provide useful insights to assist and guide planning for 4D seismic acquisition campaigns. Lastly, once field seismic data becomes available, it will assist in interpreting the observed timelapse seismic signal.

\section{Geomechanical Model Building and Simulations}

The main inputs for the grid building workflow are five geologic horizons from seismic interpretation and the reservoir model (Figure 1). The geomechanical simulation model was laterally extended by 1 reservoir length to each side to minimize boundary effects, and to allow the formation of an extended subsidence bowl. The geomechanical grid covers an area of $56 \times 71 \mathrm{~km}$, i.e., approximately $4000 \mathrm{~km}^{2}$. Vertically the model is limited between the seabed (at around $2300 \mathrm{~m}$ depth below mean sea level) and $8500 \mathrm{~m}$ depth. Vertical extent of the model is therefore $\approx 6200 \mathrm{~m}$ (Figure 2). Reservoir cells in the geomechanical grid have the same size as the original flow simulation grid. Outside the reservoir, the cell sizes were coarsened using a geometric progression in the geomechanical model up to the boundaries of the mesh. The total number of elements in the FE geomechanical model is approximately 10.2 million, of which 231.2 thousand are reservoir cells.

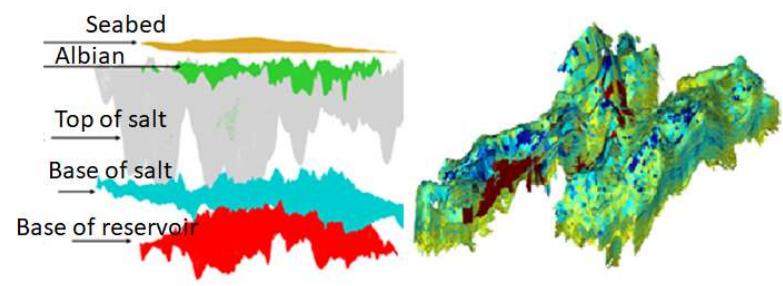

Figure 1: Images in different scales, for the regional horizons from seismic interpretation (left) and for reservoir grid with properties (right).

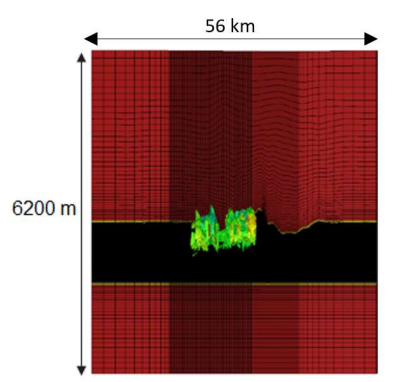

Lateral View

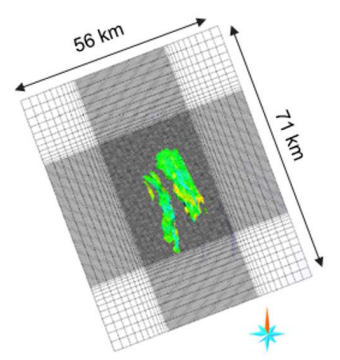

Top View
Figure 2: Dimensions of the final geomechanical grid in a lateral view (left) and top view (right).

Based on the seismic horizons, we created stratigraphic regions, assigning constant values for static Young modulus $(\boldsymbol{E})$, Poisson ratio $(\boldsymbol{v})$, and bulk density $(\boldsymbol{\rho})$. For the reservoir, the mechanical properties are: $\boldsymbol{v}=0.30$ $\boldsymbol{E}=37.0 \mathrm{GPa}$ and $\boldsymbol{\rho}=2.60 \mathrm{~g} / \mathrm{cm}^{3}$. The salt layer was assumed as homogeneous (halite). In terms of mechanical properties, we defined two scenarios representing the end members of salt behavior between differential stress relaxation in salt on long timescales, and elastic behavior maintaining large differential stresses over short timescales:

i) "Soft salt scenario", uses $v=0.49$ (close to the theoretical upper limit) and a high bulk modulus of $\boldsymbol{K}=$ $100.0 \mathrm{GPa}$. In so doing, the differential stress in the salt reduces to low values, mimicking the behavior of salt- 
creep over geological timescales, and the stress state calculated from using "high Poisson ratio, high bulk modulus" is similar to that calculated from more complex material models such as plastic behavior or creep behavior of salt (see e.g., Adachi et al., 2012). We then use equation 1 to calculate Young's modulus to be $\boldsymbol{E}=$ $6.0 \mathrm{GPa}$ :

$$
E=3 K(1-2 v) \text {. }
$$

Density of halite is $\rho=2.15 \mathrm{~g} / \mathrm{cm}^{3}$.

ii) "Stiff salt scenario", uses average seismic velocities for salt $\left(V_{P}=4500 \mathrm{~m} / \mathrm{s} ; V_{S}=2600 \mathrm{~m} / \mathrm{s}\right)$ in equations 2 and 3 to determine Young's modulus $\boldsymbol{E}$, and Poisson ratio $\boldsymbol{v}$. Consequently, $\boldsymbol{v}=0.25, \boldsymbol{E}=36.5 \mathrm{GPa}$. Bulk density is again $\boldsymbol{\rho}=2.15 \mathrm{~g} / \mathrm{cm}^{3}$.

$$
\begin{gathered}
v=1-\frac{1}{2-2\left(\frac{V_{S}}{V_{P}}\right)^{2}} \\
E=2 \rho(1+v) V_{S}^{2}
\end{gathered}
$$

The 3D elastic geomechanical simulations on a field-scale model of two producing pre-salt reservoirs are performed using Petrobras in-house software based on the finite element method. For stress field initialization for both scenarios, we use the high Poisson ratio salt. In so doing, the behavior of salt to decrease high differential stress over geologic time can be modelled without invoking timeconsuming creep calculations which are currently hard to simulate on 3D grids of the size required in this project. Production induced stress changes are then added to the initial stress field. The effect of pore-pressure depletion on the simulated stress field is included using one-way coupling between the reservoir simulation model and the geomechanical model. In so doing, the stress state in the entire geomechanical model at the times of base- and monitor seismic survey is derived. Additionally, the displacements of each node, and the strain tensor in each cell between these two timesteps are captured. The pressure depletion in the reservoir varies laterally from close to zero to more than $300 \mathrm{kgf} / \mathrm{cm}^{2}(\approx 30 \mathrm{MPa}$ or 4350 psi) locally around key wells.

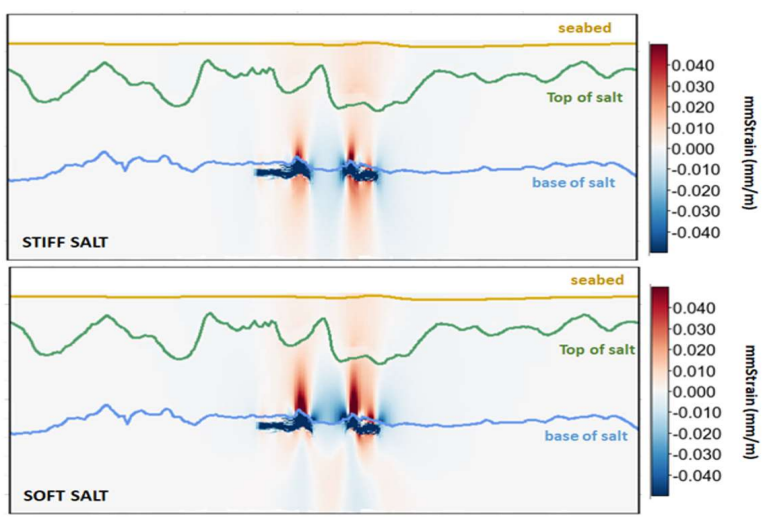

Figure 3: Vertical strains $(\mathrm{mm} / \mathrm{m})$ for stiff salt (top) and soft salt (bottom) scenarios over 22 years of reservoir production.
The geomechanical simulation results in terms of vertical strains are shown in Figure 3. Positive values (red) indicate rock dilation and negative values (blue) indicate rock compression. Note, that the color-scale is clipped to make small strain visible. Maximum dilation is $0.61 \mathrm{~mm} / \mathrm{m}$ and minimum dilation is $-0.25 \mathrm{~mm} / \mathrm{m}$. Both scenarios show an extension of the overburden, with vertical strain decreasing from the top of reservoir to the seabed. For the stiff salt scenario, the vertical strain in the underburden has comparable magnitudes to those above the reservoir. For the soft salt, the magnitudes of dilation and compression above the reservoir are greater than those in the underburden. Also note that the "soft salt" scenario produces a complex spatial pattern in the underburden, whereby dilation and compression are no longer easily correlated to pressure depletion in the reservoir.

\section{Time-shifts forward modeling: vertical propagation}

We first compute the change in compressional velocity based on vertical strains with equation (4) as proposed by Hatchell and Bourne (2005) and Røste et al. (2007):

$$
d V_{P_{\text {geom }}}=-\left(R \varepsilon_{z}\right) V_{P} .
$$

There is a large uncertainty concerning the magnitude of $R$ values and their spatial variability. For this exercise, we adopted values ranging from 2 to 10 which is consistent with observed values in many fields worldwide (Herwanger and Koutsabeloulis, 2011; MacBeth et al., 2019). The large uncertainty associated to $R$-factor values is also found by Mello et al. (2021) - submitted to $17^{\text {th }}$ CISBGF - through laboratory measurements in rock samples from Brazilian offshore basins. Note that the choice of R-factor values directly affects the simulated time-lapse seismic data and the time-shift magnitudes derived from these simulations.

We then calculate the theoretical time-lapse timeshifts due to geomechanics (neglecting the effect fluid changes in the reservoir) assuming vertical ray-paths using equation (5), where $z$ is depth and $d z$ is the thickness of each cell of the geomechanical grid:

$$
\Delta t_{\text {geom }}=2 \int_{0}^{z}\left(\frac{1}{V_{P_{\text {geom }}}}-\frac{1}{V_{p}}\right) d z
$$

In this notation, positive timeshifts indicate an increase in travel-time from base- to monitor survey. The timeshifts calculated at each location can now be applied to warp a field dataset to create a synthetic monitor survey. To create a more realistic amplitude dataset, we also add coherent and random noise to the monitor survey amplitude data. We define the coherent noise as a linear function, where its values increase with travel-time. Random noise is assumed to display a Gaussian distribution. The same noise was applied to soft and stiff salt scenarios allowing a fair comparison between both results. Because the baseline scenario is an observed field seismic data set and is intrinsically affected by noise, we decided to add the synthetic noise only to the monitor data.

We use the normalized root mean square (NRMS) to measure the synthetic $4 \mathrm{D}$ repeatability and ascertain that our synthetic noise is representative for noise encountered 
in modern high-quality 4D OBN seismic surveys - the standard acquisition technologies for high-quality seismic monitoring in the pre-salt context. For the NRMS calculation we choose a deep and a shallow $1.0 \mathrm{~s}$ length window (Figure 4). The deep window comprises the level where we expect the main 4D effects. The average NRMS is around $5 \%$ for random noise only and $8 \%$ for random plus coherent noise (Figure 5).

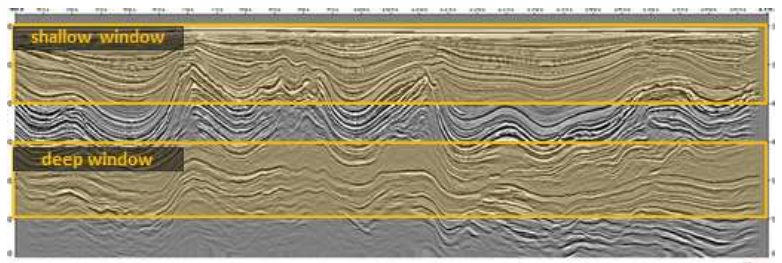

Figure 4: Seismic section across the reservoir with shallow and deep time windows used for the NRMS computation.

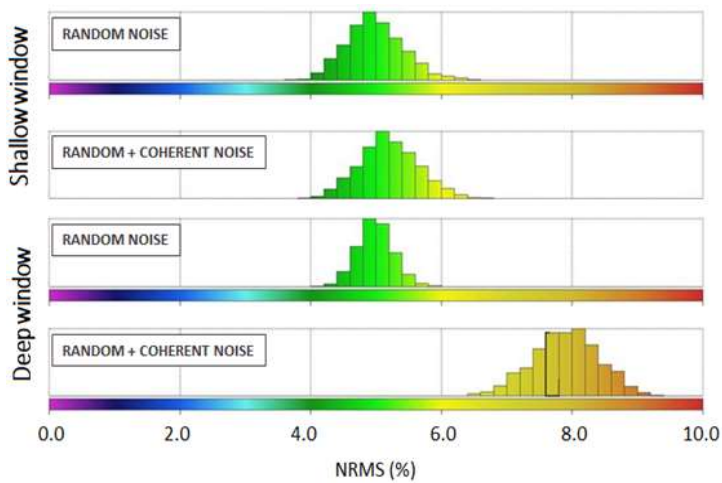

Figure 5: Final NRMS (\%) for random noise and random plus coherent noise within the shallow (top) and deep (bottom) time windows.

We can now address our main question: "Is it feasible to detect the expected magnitude of time-shifts induced by production in stiff carbonate reservoirs?". Figure 6 shows that the time-shifts at the top of the reservoir due to overburden dilation are less than $0.5 \mathrm{~ms}$. Compression effects caused by stress arching are much smaller, limited to approximately $-50 \mu \mathrm{s}$. For the "soft salt" scenario, the stress arching is more pronounced as the salt spreads the stress change and strain over a wider areal extent and causes stronger reservoir compaction and overburden dilation. This means greater positive time-shifts above the center of the depleting reservoir and greater negative timeshifts on the flanks of the reservoir (Figure 7).

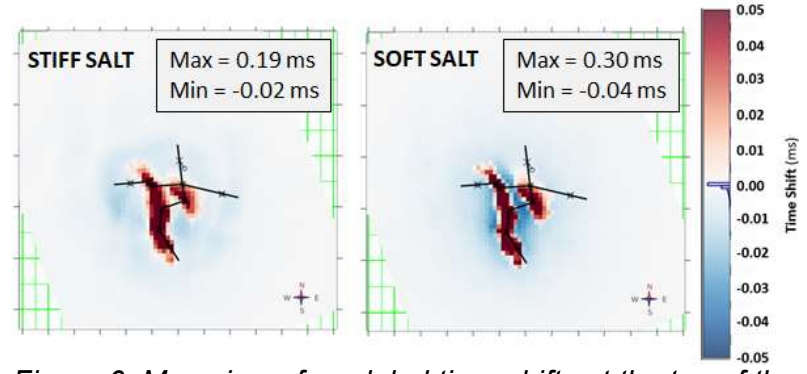

Figure 6: Map view of modeled time-shifts at the top of the reservoir for stiff salt (left) and soft salt (right) scenarios.

\section{Time-shifts estimation: vertical propagation}

Several examples of interpretable time-shift below seismic sampling are summarized in MacBeth et al., (2019). Production-induced time-shifts smaller than $1 \mathrm{~ms}$ are reported at reservoir level or overburden in seven fields, including a post-salt carbonate in the Campos Basin. The smallest timeshifts that could unequivocally be classified as reservoir production-induced signal and not as noise was of the order of $0.2 \mathrm{~ms}$. The expected overburden timeshifts for pre-salt reservoirs of approximately $0.5 \mathrm{~ms}$ should therefore be measurable, if highly repeatable seismic data are acquired and suitable care is taken during seismic processing. We find that, using a cross-correlation method, we are able to recover such small time-shifts from seismic amplitudes with good accuracy for both salt scenarios (Figure 7). This is also true even for seismic data with moderate repeatability when considering seabed acquisitions. Due to the nature of the phenomena, timeshifts caused by overburden dilation (or compression) are low frequency and often more consistent laterally and vertically compared to timeshifts measured across the reservoir level.

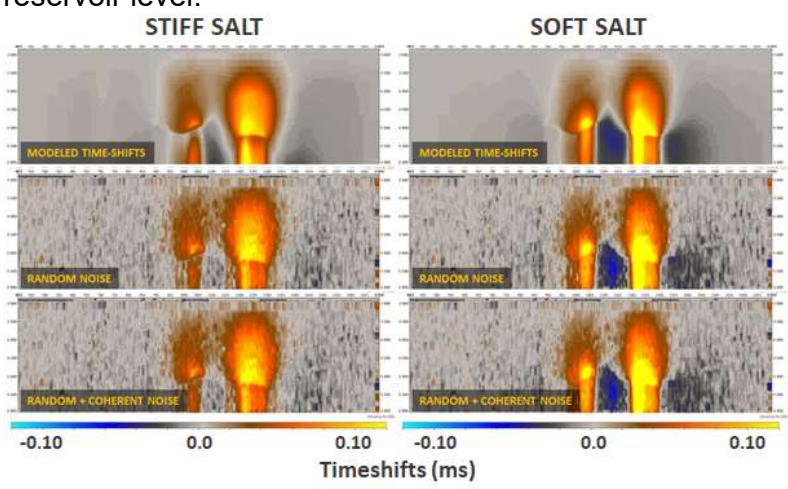

Figure 7: True time-shifts (top) and measured time-shifts using cross-correlation for the random noise case (middle) and random plus coherent noise case (bottom).

\section{Time-shifts forward modeling: 2D seismic modeling and migration}

The vertical wave propagation assumption used in the previous section is violated in the presence of large lateral velocity changes, such as created by the presence of salt with complex topography. In geological reality, wave propagation - employed in the processes of modeling and seismic imaging - is substantially more complex than assuming a $1 \mathrm{D}$ velocity model. We therefore designed a numerical experiment which mimics the major elements of a permanent reservoir monitoring system, including forward modelling of the seismic signals recorded in this geometry, and the subsequent data-processing. This type of experiment is widely recognized as a powerful tool to support acquisition parametrization and guarantee the fidelity of seismic images (e.g., Oppert et al., 2017).

The workflow followed here starts with the calculation of the monitor velocity field by updating the baseline velocity field through the simulated strains in the R-factor rockphysics model. We then compute seismic data for sourcereceivers along a $2 \mathrm{D}$ line for base- and monitor survey. The 
simulated acquisition design uses 801 receivers at the seafloor and sources at $7 \mathrm{~m}$ depth with intervals of $100 \mathrm{~m}$ and $20 \mathrm{~m}$, respectively. The maximum source-receiver offset is $8 \mathrm{~km}$, the record length is $8.0 \mathrm{~s}$ and we use a Ricker wavelet with the peak frequency of $60 \mathrm{~Hz}$. The final seismic images, for the base- and monitor from each salt scenario were generated by a Least-squares reverse time migration (LSRTM) algorithm to: (i) increase the resolution; (ii) compensate for irregular seismic illumination, and (iii) reduce migration artifacts. Finally, we estimate the timelapse timeshifts using cross-correlation between the migrated seismic images.

The seismic velocity variations induced by the rock strain are subtle: for the "soft salt" scenario the range of velocity changes is -5.69 to $+3.97 \mathrm{~m} / \mathrm{s}$ and for the "stiff salt" layer 2.77 to $+3.90 \mathrm{~m} / \mathrm{s}$. The ability to capture the influence of minute velocity variations in seismic experiments is due to their extent over for a large portion of the model, and this results in minute yet observable $4 \mathrm{D}$ signals, in the form of differences in travel-times in the imaged reflectors. Figure 8 depicts the final image for the baseline scenario and a zoom in the reservoir region showing the difference (without warping) between the stiff monitor and the baseline data.

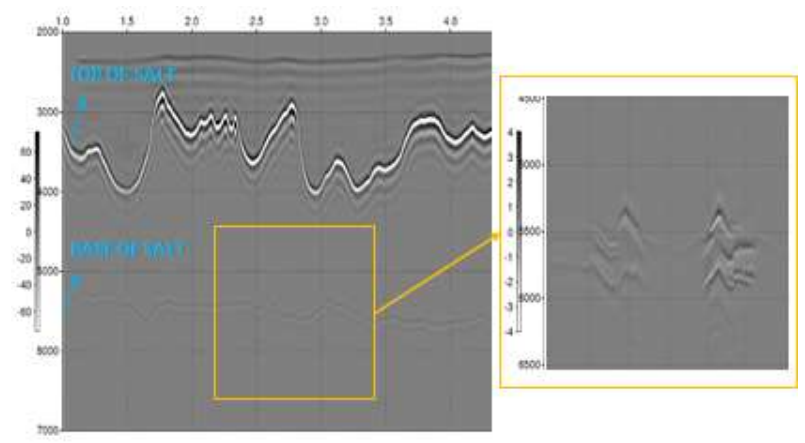

Figure 8: Final migrated LSRTM section and a zoom in from the $4 D$ difference of the stiff salt scenario.

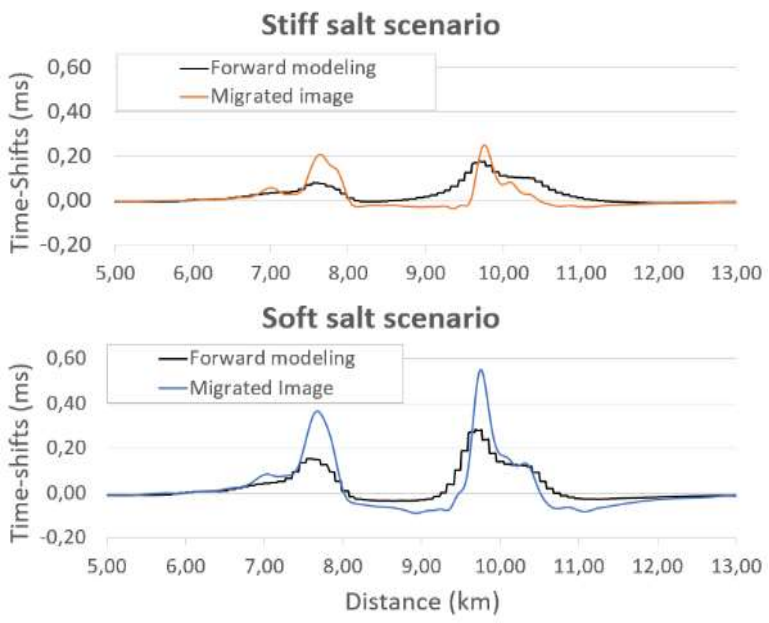

Figure 9: Comparison between time-shifts estimated from $1 D$ forward modeling (black) and from synthetic migrated images (orange and blue) along the base of salt.
The results of 1D cross-correlation between the migrated images reinforces that tiny timeshifts can be recovered for sub-salt reservoirs. Figure 9 show the timeshifts along the base of the salt in a section across the center of the field. There are two anomalies approximately $2 \mathrm{~km}$ apart, located right above the main depletion areas. For the "stiff salt" scenario, the expected magnitude of time-shifts at these two anomalies are less than $0.2 \mathrm{~ms}$ and $0.1 \mathrm{~ms}$. The measured timeshifts from migrated images overestimates the expected timeshifts from 1D modeling, but clearly delimit the 4D anomalies. For the "soft salt" scenario, the trend of time-shifts along the horizon is well captured, with some local overestimates. The (weak) stress-arching effect causing negative timeshifts of $-0.05 \mathrm{~ms}$ is also captured. The source of the difference between expected timeshifts from 1D modelling and measured timeshifts after migration have been previously observed and explained by Cox and Hatchell (2008), as an effect of reservoir topography and slightly different ray-paths between base- and monitor survey.

\section{Future work}

Building a heterogeneous model from integration of different sources of data and scales (laboratory measurements, well-log data, and field scale geomechanical and seismic models) will provide a more realistic subsurface representation. This integration is important to derive results that are more descriptive of the geological reality. To make further studies even more relevant to time-lapse seismic campaigns it is necessary to investigate the impact of different acquisition geometries, as well as including source and receiver repeatability into the simulations. This is work in progress and will take advantage of a combination of 2D LSRTM exercises covering realistic imaging issues and a ray-tracing scheme to rapidly access time-shifts derived from $3 \mathrm{D}$ propagation, as presented by Dias et al. 2021 (submitted to $17^{\text {th }}$ CISBGF).

\section{Conclusions}

Our work suggests that time-lapse seismic can be used to observe geomechanically induced timeshifts in strongly depleted stiff carbonates reservoirs under complex salt layers. The simulations also suggest that highly repeatable and good quality 4D seismic data will be required. The expected magnitude of timeshifts is possibly at the lower end of detectability. There are several factors why observations from field data may be larger than those predicted from the simulations. Firstly, displacements and strains are, among other factors, a direct consequence of reservoir compressibility. The chosen values represent compressibility of a stiff carbonate rock matrix, and do not account for soft inclusions such as karsts features and fractures. These features are expected to make overall reservoir compressibility higher and the actual geomechanical effects stronger as well as increasing the expected 4D response. On the other hand, the high uncertainty on rock velocity-strain relations cannot be ignored and the hypothesis of a more challenging scenario in terms of timeshifts detectability must be considered.

There are substantial differences in the overburden response between the two simulated scenarios. In the case 
of "soft salt", the salt takes up most of the reservoir compaction, and there is hardly any underburden movement. In the case of "stiff salt", the reservoir compaction splits evenly between overburden (salt) and underburden. Currently, there is not sufficient experimental evidence to know which way salt behaves over production timeframes, and how this will affect the observed 4D response. With experience of field production and observations of the geomechanical overburden response, we will re-assess the material model for salt (including the need for using plasticity or visco-elasto-plasticity). Another major uncertainty presents itself in the way wave speeds in salt react to production induced deformation of the various salt lithologies.

With our current knowledge, we note that for the "soft-salt" scenario the stress-arching phenomena is more pronounced, and timeshifts decay faster through the salt with distance from the reservoir than for the "stiff-salt" scenario. The most striking difference between the two scenarios is the width of the time-lapse timeshift anomaly at top of salt and base of salt, with the "stiff-salt" scenario spreading the timeshift anomaly over a wider footprint. Since both top-of-salt and base-of-salt are strong reflectors, any observations of time-lapse time-shifts at topof-salt and base-of-salt should give valuable information on bulk salt behavior during reservoir production.

To conclude: the presented results show that current acquisition technology and existing seismic processing and analysis algorithms should be able to successfully recover the expected geomechanical signals (i.e., timelapse timeshifts). Both the shape and magnitude of these 4D anomalies can be measured quantitatively with sufficient accuracy and will require sub-sample precision determination. Undoubtedly, once field time-lapse seismic data become available, we will encounter some observations which were not anticipated. This is to be expected, as seismic monitoring of pre-salt carbonate reservoirs is still in its early stages. Historic experience shows that unexpected behavior typically leads to an improved understanding of the associated geomechanical and seismic processes and will ultimately lead to better field management decisions.

\section{Acknowledgments}

The authors thank PETROBRAS for authorizing this publication, TOTAL and our colleagues Mario Paes, Carlos Eduardo Pereira, Josenilda Lonardelli, Ciro Climaco, Phillip Marchina, Tom Blanchard, Igor Escobar, Elisa Sotelino, Matheus Peres, and Leonardo Mesquita for the collaboration on this topic.

\section{References}

Adachi, J.I., Nagy, Z.R., Sayers, C.M., Smith, M.F., and Becker, D.F., 2012. Drilling Adjacent to Salt Bodies: Definition of Mud Weight Window and Pore Pressure Using Numerical Models and Fast Well Planning, SPE 159739MS. DOI: https://doi.org/10.2118/159739-MS

Cox, B., and Hatchell, P., 2008. Straightening out lateral shifts in time-lapse seismic, First Break. DOI: https://doi.org/10.3997/1365-2397.26.1119.27997
Dias B.P., Mello, V.L., Borges, F.A.S., Dias, R.M., 2021. Angle-dependent time-shift modeling by 3D ray-tracing. Submitted to the 17th International Congress of the Brazilian Geophysical Society \& Expogef.

Dusseault, M., Bruno, M.S., and Barrera, J.A., 2001 Casing Shear: Causes, Cases, Cures, SPE Drilling and Completion. DOI: https://doi.org/10.2118/72060-PA

Geertsma, J., 1973. Land Subsidence Above Compacting Oil and Gas Reservoirs. Journal of Petroleum Technology 25: 734-744. doi: https://doi.org/10.2118/3730-PA

Hatchell, P.J., and Bourne, S.J., 2005. Measuring reservoir compaction using time-lapse timeshifts: SEG Technical Program Expanded Abstracts 2005. 2500-2503. DOI: https://doi.org/10.1190/1.2148230

Herwanger, J., and Horne, S., 2009. Linking reservoir geomechanics and time-lapse seismics: Predicting anisotropic velocity changes and seismic attributes, Geophysics. DOI: https://doi.org/10.1190/1.3122407

Herwanger, J., and Koutsabeloulis, N., 2011. Seismic Geomechanics: How to build and calibrate geomechanical models using 3D and 4D seismic data, EAGE publications, ISBN number 978-90-73834-10-1

MacBeth, C., Mangriotis, M. D., \& Amini, H., 2019. Poststack 4D seismic time-shifts: Interpretation and evaluation. Geophysical Prospecting. DOI: https://doi.org/10.1111/ 1365-2478.12688

Mayuga, M. N., 1970. Geology and development of California's giant - Wilmington oil field. Geology of Giant Petroleum Fields. AAPG Memoir 14

Mello, V.L., Vasquez, G. F., Justen J.C.R., Morschbacher, M.J., Borges, F.A.S., Rossi, D.F., Amaral, R.F., Herwanger, J., Leroy, Y.M., 2021. Velocity-strain relations from hydrostatic measurements on multiple lithologies. Submitted to the $17^{\text {th }}$ International Congress of the Brazilian Geophysical Society \& Expogef.

Nagel, N.B., 2001. Compaction and subsidence issues within the petroleum industry: From Wilmington to Ekofisk and beyond, Physics and Chemistry of the Earth, Part A: Solid Earth and Geodesy, DOI: https://doi.org/10.1016/S1464-1895(01)00015-1.

Oppert, S., Stefani, J., Eakin, D., Halpert, A., Herwanger, J., Bottrill, A., Popov, P., Tan, L., Artus, V., and Oristaglio, M., 2017. Virtual time-lapse seismic monitoring using fully coupled flow and geomechanical simulations. The Leading Edge 36: 750, 752-768. DOI: https://doi.org/10.1190 /tle36090750.1.

Røste, T., Landrø, M. and Hatchell, P., 2007. Monitoring Overburden Layer Changes and Fault Movements from Time-Lapse Seismic Data: Conference Proceedings, 69th EAGE Conference and Exhibition incorporating DOI: https://doi.org/10.3997/2214-4609.201401685

Sulak, R.M., 1991. Ekofisk Field: The First 20 Years. J. Pet. Technol. DOI: https://doi.org/10.2118/20773-PA 